\title{
A Voting System for Internet Based Democracy
}

\author{
Stefan Dirnstorfer \\ Thetaris GmbH stefan@thetaris.de
}

\begin{abstract}
A lot of research has been spent on the process of decision making in large groups. While we easily find widely accepted solutions within groups of friends, the process somehow does not scale up to larger and more distant groups of individuals. Some of problems have been attributed to the multiple dilemmas known in voting theory.

This paper introduces an interactive voting game that avoids the loss of pareto optimallity and the influence of voting tactics that is based on non-public ballots cast by peer voters. At its down side it might be subject to nonconverging voting behaviour and not produce a final result within a confined time frame.
\end{abstract}

\section{The Social Dilemma}

The social dilemma, often described as the prisoners dilemma, is said to be the fundamental problem of social cooperation. If the social dilemma is present, the group decision will not be pareto optimal, due to the individuals pursuing their self interests. This section explains that the social dilemma is present in a when voting on a number of interconnected issues in a separate vote.

Any voting system that is not pareto optimal is not suitable to solve the social dilemma. Minor disputes between group members must be set aside in favor of a common goal. The examples below show that separate voting on separate decisions is not suitable for resolving social dilemmas that require the arbitration over multiple initiatives.

\section{$1.1 \quad$ Example 1}

Much thought has been spent on the procedures of a single vote on mutually exclusive choices, such as the election of a representative or the election of a political party $[1,4]$. As soon as multiple choices have to be made on separate issues democratic voting does a very bad job in satisfying the involved voters, as will be shown by the following example.

Figure 1 shows a ballot paper that could be used to conduct a vote on two separate initiatives. As it turns out, a classical voting system is often unable to produce the most satisfying result. Assume that two people participate in this vote. For simplicity they will be called Left and Right. Left is a fanatic supporter of initiative A and slightly biased against initiative B. Right is a radical fan of initiative B and somewhat biased against initiative A. Since both initiatives don't exclude each other, it is obvious that they could both be implemented with satisfying results for Left and Right. However, that is not the result of a democratic vote.

The fundamental idea behind a democratic vote is that each participant expresses their personal preferences in a selfish fashion and yet, the optimal 
Fig. 1. Ballot paper with two non-exclusive choices

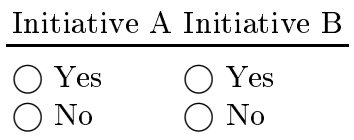

decision could be derived through a mathematical counting scheme. Under such an objective Left would vote (Yes,No) and Right would vote (No,Yes). Without cooperation neither initiative can achieve the necessary majority and the result would be (No,No), although both participants agree that (Yes,Yes) would have been a better option. This result is known in game theory as the Prisoners Dilemma [5]. Figure 2 shows the dilemma in matrix form.

Fig. 2. Social dilemma in a vote on two independent initiatives: Left would be happiest with (Yes,No), while Right favors (No,Yes). Both could be satisfied with a (Yes,Yes) result, but unarbitrated voting leads to a suboptimal (No,No) decision.

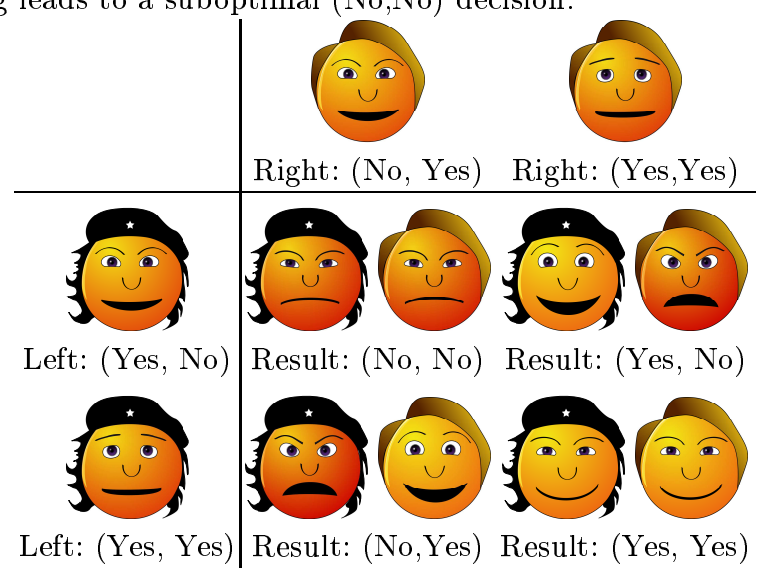

In practical politics the optimal solution requires a benevolent member of parliament who arbitrates between Left and Right. This job can only be done through a small number of delegated representatives who can build sufficient trust in each other. Internet based activist groups usually do not have the resources to sustain such arbitration efforts, or it even contrasts self selected grassroots democratic principles. With an increasing number of decisions that have to be made, more and more arbitration opportunities will be lost and group satisfaction decreases.

Looking at our simple example one might rightfully object that there must have been someone to come up with a voting scheme that elicits the interdependence between the two initiatives. Many such schemes are subsumed under the Condorcet voting system which requires the participants to assign ordinal preference numbers to the four mutually exclusive choices (No,No), (No,Yes), (Yes,No), and (Yes,Yes) as shown in figure 3. All common variations of the Condorcet vote can solve this simple problem [4]. Such overarching voting schemes 
are feasible for a small number of clearly interlinked issues, but effort grows exponentially with the number of involved decisions.

Fig. 3. A Condorcet vote could solve the social dilemma for two choices, but becomes impractical for a larger number of seemingly independent decisions. Choices for initiatives A, B Left's preferences Right's preferences

$\begin{array}{ccc}\text { (No, No) } & \text { 3rd } & 3 \text { rd } \\ \text { (No, Yes) } & 4 \text { th } & 1 \text { st } \\ \text { (Yes, No) } & 1 s t & 4 \text { th } \\ \text { (Yes, Yes) } & \text { 2nd } & \text { 2nd }\end{array}$

\section{$1.2 \quad$ Example 2}

This second example will again demonstrate that democratic voting does not automatically reach the pareto optimal solution for the group, if it is applied separately on seemingly unconnected issues. This time initiative A and B are not technically excluding each other, but they compete for some sort of limited resource that makes in impractical to implement both, A and B.

Fig. 4. A possible vote distribution for two choices that are not strictly exclusive but unduly strain group resources, when combined.

\begin{tabular}{l|cr} 
& pro A contra A \\
\hline pro B & $10 \%$ & $45 \%$ \\
contra B & $45 \%$ & $0 \%$
\end{tabular}

Suppose two initiatives, A and B, are completely independent in terms of phenomenological effect, but both deplete the group's limited financial or natural resources. Only $10 \%$ of the electorate think that it makes sense to implement both measures, while $90 \%$ think that only one of the suggested measures can be implemented in a sustainable fashion. Yet, under these tight conditions separated democratic voting can lead to exactly this highly unsatisfactory outcome for the group. Figure 4 shows the distribution of voters and figure 5 shows the results.

Fig. 5. Separated voting favors both initiatives. Only $10 \%$ are satisfied, while $90 \%$ complain about wasted resources.

\begin{tabular}{l|ll} 
& pro contra \\
\hline Initiative A & $55 \%$ & $45 \%$ \\
Initiative B & $55 \%$ & $45 \%$
\end{tabular}


If such a democratic system with independent votes on independent political issues governs a political state at least a financial solution can be found easily: Charge future generation. However, for small groups as well as for profit oriented businesses this is not an option. That is why grassroots democratic principles are never found in the industry on any significant scale and it is the reason, why grassroots organizations always break apart quickly.

\section{The Internet Voting System}

The internet voting system is a voting system primarily designed to help solving the social dilemma. It is to be played interactively and requires all votes to be publicly viewable during the voting phase.

Since conflicting positions can only be resolved by arbitrations over multiple initiatives, the voting system must consider all open decisions simultaneously. Thus, a huge number of potential outcomes have to be evaluated, which is only possible by utilizing the unique features of an interactive computational platform without the restrictions of a physical ballot sheet. Hence, the internet voting system is specifically designed for the internet and is not applicable to paper based voting.

The internet voting system is more akin to cooperative and interactive game play than to classical voting. Many strategy games prove that it is possible to take simultaneous and fine grained judgments in a huge number of political fields. This potential must be exploited to find widely agreed preference orders. The results will be more fuzzy in terms of absolute valuations, but more agreeable and consistent in terms of its result. At its downside it does not enforce a final winner if the voters preference orders are circular.

\section{$2.1 \quad$ Vote Counting}

The internet vote counting scheme follows the physical model of mechanical forces. Different spacial directions correspond to political initiatives that can be supported or opposed. All users of the system can pull with equal force into any direction that corresponds to their mix of political convictions. Just as in physics transverse forces cancel each other, while correlated forces add to each other.

We can represent the voting behavior as a matrix $V \in \mathbb{R}^{m \times n}$. The entries $\mathrm{v}_{\mathrm{pi}}$ refer to the voting weight assigned by participant $p$ to initiative $i$. The weights $v_{p i} \in \mathbb{R}$ can be positive to express support and negative to express opposition. The total voting weight assigned by a participant is confined to length one of the resulting force vector $v_{p}$ in Euclidean space. Hence, the summed squared voting weights are confined to one.

$$
\left\|v_{p}\right\|^{2}=\sum_{i=1}^{n}\left(v_{p i}\right)^{2}=1
$$

The resulting group preference $\mathrm{R}(\mathrm{i})$ for an initiative i can be obtained as the sum of all directional forces applied in the i-th component of all participant's 
voting vectors.

$$
R(i)=\sum_{p=1}^{m} v_{p i}
$$

Fig. 6. Comparison of possible vote distributions in different voting systems. A marker shows an example for voting weights that could be allocated in a vote on two initiatives.

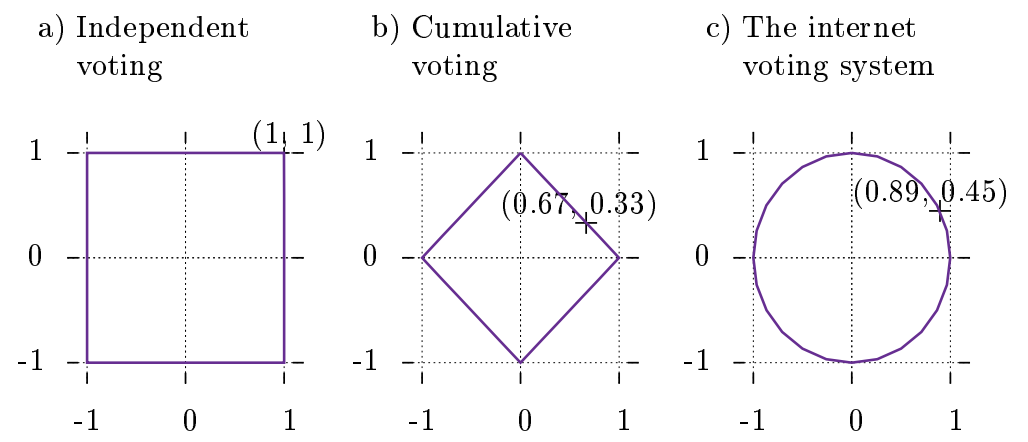

Figure 6 compares the possible vote distributions of different voting systems in a vote with two initiatives. The voting weight assigned to the first initiative is plotted on the $\mathrm{x}$-axis, while the second weight is plotted against the $\mathrm{y}$-axis. All achievable vote combinations are shown in the graph.

a) If participants can vote on both initiatives independently an associated voting weight between -1 and 1 can occur on both axes. If such a voting system is applied to non-political topics, such as customer satisfaction, it is usually possible to assign fractional weights. In a political struggle, however, hardly anyone would deliberately limit their potential influence. Hence, slight convictions for any side of the debate leads to a full vote assigned in the corresponding direction.

b) In a cumulative voting system participants can distribute a limited total voting weight onto different initiatives. Under such a voting system it can make sense to split a vote onto different initiatives. If, however, one initiative is seen as considerable more important than the other, participants would hardly split votes at all. Why give up a fraction of a vote in a major issue, when one can just gain the same fraction in a minor issue.

c) The internet voting system uses a limited radial voting weight. The system allows maximum total influence, if the vote is spread evenly on both initiatives. At the same time, the system allows higher influence on one initiative, if more weight is assigned to it. As discussed in more detail below, this is the only voting system that makes it optimal to correctly reveal relative preferences.

Figure 7 shows a possible voting strategy. Four participants have contrary positions on two initiatives. P favors (Yes,Yes), Q (No,Yes), R (No,No), and $\mathrm{S}$ wants (Yes,No). Due to different priorities assigned by each participant the resulting sum has a slight tendency towards (no,no). As will be discussed in more detail, any two participants can overcome the social dilemma and cooperate to 
Fig. 7. Undecidable vote with four participants and two initiatives. Cooperation can increase influence. In the right graph, $\mathrm{P}$ and $\mathrm{S}$ express their cooperative instead of their individual preferences.

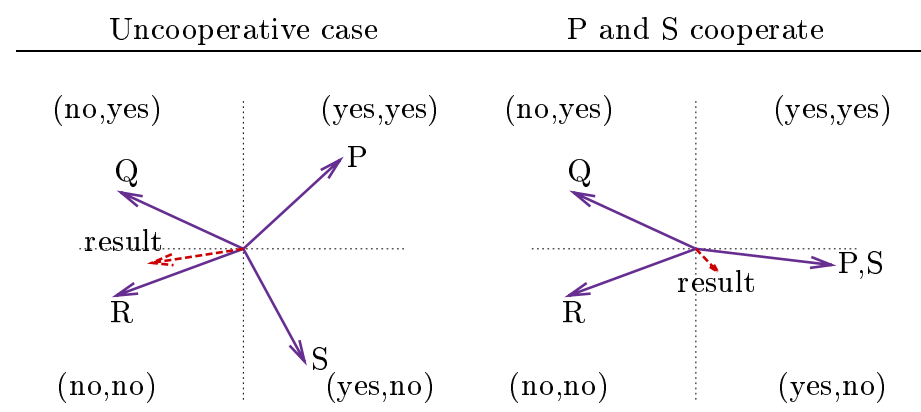

increase their influence on the result. While cooperating each group member votes according to group preferences. Here, $\mathrm{R}$ and $\mathrm{S}$ push the result slightly towards a (Yes,No).

\subsection{Vote Delegation}

Vote delegation is often seen as a way to hand over responsibility to someone who is trusted to make the most informed decisions. The internet voting system allows vote delegation to a number of different delegates, whose consenting positions are expressed as the delegators vote, while dissenting positions are abstained from. Furthermore, circular votes delegations are allowed. Hence, delegated votes are not necessarily passed one way upstream, but the can circulate within a group and ensure a certain degree of cooperative voting.

A Participant $p$ can delegate a portion $\mathrm{d}_{\mathrm{pq}}$ of her voting weight to participant $q$ and change her vote from $v_{p}$ to an average with $v_{q}$. The vote delegation mechanism then computes a transformed vote vector $\tilde{v}_{p}$ which is expressed as the new vote vector and which is counted in the evaluation of the total result.

$$
\tilde{v}_{p}=n\left(v_{p}+\sum_{q} d_{p q} * \tilde{v}_{q}\right)
$$

Function $n$ is a vector normalization, that ensures full voting activity, even if delegates disagree on any of the initiatives.

$$
n(v)=\frac{v}{\|v\|}
$$

Technically speaking vote delegation exchanges ones own vote vector with a weighted combination of delegate vote vectors. From a gaming perspective this has significant consequences. Delegations are public expressions of cooperative intentions, but they are no proofs that cooperation in maintained. In fact, the cooperative behavior can not be observed and each group member can defect into voting along her initial preferences. This leads to the social dilemma as shown below, with the special feature that cooperative opportunities exist between almost any two participants. 


\section{Utilitarian Considerations}

The utilitarian approach provides a mathematical framework for the behavior of rational individuals [2], which allows us to derive a number of strategic properties of the discussed voting system. We consider a utility function $u_{p}$ that measures the satisfaction that participant $p$ gets from the results. Naturally, $p$ wants to maximize her utility and, assuming rational behavior, votes accordingly.

$$
v_{p}=\operatorname{argmax} u_{p}(R(1), R(2), \cdots, R(n))
$$

In the following, it must be assumed that the utility function $u_{p}$ is smooth and without local extrema. Smoothness can be derived from the fact that no sudden or discontinuous decisions can be taken from the system. All results are either of advisory nature, or are run through a slow and smooth approval process. The lack of local extrema implies that all participants always want to change the political landscape into any direction and are never fully satisfied with the status quo.

\subsection{Optimal Voting Weights Correspond to Real Preferences}

The first property teaches us that it is optimal to allocate voting weights according to real preferences. Hence, the ratios of optimal voting weights equal the ratios of real subjective preferences. These can be expressed as the utility gradient $\nabla u_{p}$ that points in the direction steepest ascent, i.e. the direction with quickest gain in utility. It will be shown that the voting vector $v_{p}$ points in the same direction, but with a normalized length. Hence, a vote $v_{p}$ reveals a first order approximation of $p$ 's utility function.

$$
v_{p}=n\left(\nabla u_{p}\right)=\frac{\nabla u_{p}}{\left\|\nabla u_{p}\right\|}
$$

Proof: Let $x_{r}$ be the total result of the vote $x_{r}=\sum_{q} v_{q}$ and $x_{0}$ be the result prior to the participation of $p$, i.e. $x_{0}=x_{r}-v_{p}$. The contribution of $p$ can adjust the result within the constraints provided by the voting system $\phi=\left\|x_{r}-x_{0}\right\|-1$ where $\phi\left(x_{r}\right)=0$. Now, $p$ wants to maximize her utility with subject to above constraints. By the method of Lagrange multipliers we can conclude that utility maximization is achieved when $\exists \lambda: \nabla u=\lambda \nabla \phi$, whereas $\nabla \phi=2\left(x_{r}-x_{0}\right)=2 v_{p}$ and finally $\lambda=\|\nabla u\| / 2$.

While few people will be able to specify their marginal utility with such an accuracy it is never the less important that utility can not be increased by voting against ones personal preferences. Second, accurate preference vectors can be derived from averages over a number of delegated group members.

\subsection{The First Marginal Vote has Zero Cost}

The second property confirms that the first marginal voting weight comes for free. Hence, it is just rational to assign some voting weight to an initiative at ones 
slightest conviction. It does not require noticeable reduction of voting power on other initiatives.

This property also has strong implications for cooperative voting strategies. Any topic you feel almost indifferent about can serve as a valuable asset in a vote swapping deal. Such deals are extremely important in the quest for solving the social dilemma. Naturally, you would prefer to enter such a cooperative deal with someone who has matching core principles.

Assuming participant $p$ intends to allocate some voting weight to a new initiative $i$. Due to the limited overall voting weight $p$ has to withdraw some votes from any other initiative. Here, she decides to cut back on $j$. The question now is how much weight must be subtracted from $j$ in order to increase her vote on $i$ by one marginal unit. As indicated previously the answer is zero.

$$
\lim _{v_{p i} \rightarrow 0} \frac{d v_{p j}}{d v_{p i}}=0
$$

Proof: The voting weight is confined by $\left\|v_{p}\right\|^{2}-1=0$. Building the total differential yields $\sum 2 v_{p i} d v_{p i}=0$. Division by $d_{p i}$ yields $d v_{p j} / d v_{p i}=-v_{p i} / v_{p j}$.

The high exchange value of the first marginal vote is consistent with real world observations. Few seconds spent on signing a petition are met with hours of campaigning efforts, while a full time commitment to a political movement hardly pays the bills. Dedicated interest groups also profit from the large status gained by representing their members in specialized issues.

\subsection{Any Two Participants Can Cooperate}

The third property concerns the ability to increase influence through cooperative voting. Joint voting tactics are known to prevail in all democratic voting systems [3]. Often it is not easy to find such opportunities, since they might occur only between certain participants with a suitable preference match. In this respect the internet voting system is much more democratic. Any two participants can cooperate, almost regardless of their political preferences.

The internet voting system is designed as an interactive game, where votes can be viewed and reassigned. Tactical voting is an intended part of the system. The internet voting system does not try to resolve pathological cases with circular and twisted preference orders. It just shows the presence of such inconsistencies as a sequence of non-converging voting results. If cyclic voting behavior was to be resolved, more negotiations have to take place and new initiatives must be added to serve as a potential incentive for arbitration.

In the most simple cooperative situation between two participants $p$ and $q$ each side delegates a positive voting weight $\epsilon$. The symmetric delegation is certainly not the only cooperative option. There might be a number of reasons why the other side should delegate a larger voting fraction. Anyway, for the following discussion $p$ delegates to $q$ with $d_{p q}=\epsilon$ and vice versa $d_{q p}=\epsilon$. Following equation (3) new voting vectors $\tilde{v}_{p}$ and $\tilde{v}_{q}$ can be derived.

$$
\tilde{v}_{p}=\frac{v_{p}+\epsilon v_{q}}{\left\|v_{p}+\epsilon v_{q}\right\|}, \tilde{v}_{q}=\frac{v_{q}+\epsilon v_{p}}{\left\|v_{q}+\epsilon v_{p}\right\|}
$$


This mutual delegation is almost always beneficial for both sides. There are only two exceptions. First, $p$ and $q$ already have equal opinions and equal preferences on all initiatives, i.e. $v_{p}=v_{q}$. Maximum cooperation is achieved. It is not possible to cooperate further. Second, $p$ and $q$ have exactly opposing preferences $v_{p}=-v_{q}$. Both sides disagree on all initiatives with equal preference weight. Excluding these two special cases there always exists a delegation weight $\epsilon$ for which both participants can increase their utility.

$$
\begin{aligned}
\exists \epsilon>0: u_{p}\left(\tilde{v}_{p}+\tilde{v}_{q}\right) & >u_{p}\left(v_{p}+v_{q}\right) \wedge \\
u_{q}\left(\tilde{v}_{p}+\tilde{v}_{q}\right) & >u_{q}\left(v_{p}+v_{q}\right)
\end{aligned}
$$

Proof: We start with building the derivative of the normalizing denominator: $\frac{d}{d \epsilon}\left\|v_{p}+\epsilon v_{q}\right\|=\sqrt{\left(v_{p}+\epsilon v_{q}\right) \cdot\left(v_{p}+\epsilon v_{q}\right)}=1 / 2 \sqrt{\|\cdot\|}\left(v_{q} \cdot\left(v_{p}+\epsilon v_{q}\right)+\left(v_{p}+\epsilon v_{q}\right) \cdot v_{q}\right.$. Inserting an initial $\epsilon=0$ yields $v_{p} \cdot v_{q}$

No we can continue to construct the derivative of $u_{P}$ at $\epsilon=0 . \frac{d}{d \epsilon} u_{p}\left(n\left(v_{p}+\right.\right.$ $\left.\left.\epsilon v_{q}\right)+n\left(v_{q}+\epsilon v_{p}\right)\right)=\left(\nabla u_{p}\right) \cdot\left(v_{q}-v_{p}\left(v_{p} \cdot v_{q}\right)+v_{p}-v_{q}\left(v_{p} \cdot v_{q}\right)\right)$. Inserting $\nabla u_{p}=v_{p}$ and $v_{p} \cdot v_{p}=1$ yields $1-\left(v_{p} \cdot v_{q}\right)^{2}$, which is positive for $v_{p} \neq \pm v_{q}$.

Joining a cooperative formation group members do no longer vote along their personal preferences. Instead, they must strive to optimize group utilities with $n\left(\nabla \tilde{u}_{p}\right)=\tilde{v}_{p}$ and $n\left(\nabla \tilde{u}_{q}\right)=\tilde{v}_{q}$. If one side fails to do so cooperation breaks. Because the global existence of $\tilde{u}_{p}$ and $\tilde{u}_{q}$ is not guaranteed, building consistent and sustainable cooperation remains a challenge.

It is important to realize that temporary cooperation can occur even between participants who disagree on all issues. As soon as absolute preferences deviate each side can gain support in an important area, in exchange for giving up what is perceived as a less important issue.

Now it is time to revisit our initial example from section 1.2. Two large fractions disagree on all two initiatives with (Yes,No) against (No,Yes). In a disconnected vote it is up to a small minority to decide for an arbitrary outcome. In the suggested voting system this is not the case. The two large groups can cooperate temporarily and favor a (No,No) over a (Yes, Yes), just by putting a little more preference on opposing the other initiative than on supporting the own. Assuming minimal cooperative intentions the minority group is only left with joining either of the larger fractions. It can not open up a third (Yes,Yes) path.

\subsection{Cooperation Leads to the Social Dilemma}

The fourth property reminds us that the suggested voting system does not automatically resolve the social dilemma. It can only help doing so and support the required negotiations. The cooperative state is not a Nash equilibrium. It still takes mutual social links and a fundamental will to work together.

The political consequences of a failed cooperative state are often severe. Two parties first agree to cooperate and start implementing a joint set of initiatives. But then, cooperation breaks and each side denies necessary amendments to initiatives they originally supported. The results are incomplete and inconsistent laws that don't match the intended spirit. 
The internet voting system bears a similar dilemma. Despite the fact that voting vectors are permanently visible, the cooperative status can not be objectively determined. This is due to an abundance of non-defective reasons why the voting vector can change. With a large number of excuses at hand one does not need to fear immediate retaliation when defecting in a cooperative vote.

Delegation weights can publicly announce cooperative intentions, but neither their existence nor their absence bears any proof. The effect could as well have been replicated manually, or undone with the remaining voting weights.

The observation of the voting vectors can also be misleading. If a participant restores her voting vector to a precooperative state it can have many reasons. For one, her preferences might have changed to a more extreme position and she now votes what she believes to be the fair compromise. Another reason might be that she entered a cooperation with an even more extreme participant. Her cooperative influence is then visible in the third participant's vote.

Even an unchanged voting vector does not necessarily proof unchanged cooperative intentions. Maybe the participant displayed an exaggerated position prior to the cooperative deal and would have voted for what she pretends to be a cooperative concession anyway.

Figure 8 shows an example of a cooperative dilemma. Both participants agree on the first initiative, but disagree on the second. Since their voting power on the second initiative cancels out anyway, they could as well focus entirely on the first initiative. Whichever side manages to get away with a defective vote can get the best results.

Fig. 8. Social dilemma in cooperative voting. $\mathrm{P}$ has a utility of $u_{p}=R(1)+R(2)$ while Q's utility is $u_{q}=R(1)-R(2)$. In the cooperative state both can gain a utility of $u_{p}=u_{q}=2.0$. If one side defects its utility increases to 2.41 while the other side is left with a mere 1.0.

\begin{tabular}{l|cc} 
& Q: $(0.71,-0.71) \mathrm{Q}:(1.0,0.0)$ \\
\hline P: $(0.71,0.71)$ & $(1.41,0.0)$ & $(1.71,0.71)$ \\
P: $(1.0,0.0)$ & $(1.71,-0.71)$ & $(2.0,0.0)$
\end{tabular}

The only known solution to the social dilemma exists in a multiperiod repeated play. Therefore the internet voting system is more assistive in solving the social dilemma. Beneficial cooperative states can be upheld for a long period without the need to renegotiate after any changes in the political landscape.

The internet voting system awards the ability to cooperate even in the light of political battles with an increased influence on the final result. No matter what the outcome of the vote, these people are the ones that should have the most power. Only they can guarantee sustainable and stable policies. Given a group with low entry and exit barriers, it is fair to assume that the fraction which has higher cooperative tendencies is more adept to represent the group. Maybe it's even fair to say: they are the group. 


\section{Conclusion}

A voting system that grants full voting weight on each initiative does appeal to the political hardliners. Abstention or any indication of low preference must be seen as a weakness. The vote could have been swapped or sold for a political favor in an other area. The social and political implications are severe. Politicians must falsely display strong preferences on any issue, which then complicates arbitration efforts.

This document suggests a fixed voting system that can be used by groups of activists with good governance as a central goal. The system awards a balanced expression of political views with higher voting weight in key areas and it supports the formation of cooperating groups of voters that can span many decisions regardless of actual preferences. Thus, the voting system puts its focus on arbitration and on the solution of the social dilemma.

Harvesting the interactive features of the internet makes the suggested voting system the first of its kind. By combining elements of online games and social networks it enables a new dimension of problem solving capabilities. Each participant is represented by a voting vector. As soon as mutual consent is achieved, the vectors start pointing into the same direction. This does not automate the process of solving the social dilemma, but provides some metrics of well you did.

Democracy is often expected to provide an automated problem solving machine that turns personal preferences into the public good. In many Western democratic states this fatal misconception is so strong that it even survives military defeat and the obvious inability to solve the sustainability problem. Many fanatics are in power, who stick to a mantra of individualistic thinking leading to the public good. This mantra is recited in the awake of decay and crises on a global scale. If this document and the herein suggested voting system can play a role in a cooperative political revolution then it has served its purpose.

\section{References}

1. Michael A. Jones. The Geometry behind paradoxes of voting power. Montclair State University, 2006.

2. Alfred Marshall. Principles of Economics. London: Macmillan, 1920.

3. Philip J. Reny. Arrow's Theorem and the Gibbard-Satterthwaite Theorem: A Unified Approach. University of Chicago, 2000.

4. Warren D. Smith. Descriptions of single-winner voting systems. 2006.

5. John von Neumann and Oscar Morgenstern. Theory of Games and Economic Behavior. Princeton University Press, 1944. 